\title{
Effects of sodium bicarbonate supplementation on exercise performance: an umbrella review
}

\author{
Jozo Grgic ${ }^{1 *} \mathbb{D}$, Ivana Grgic ${ }^{2}$, Juan Del Coso $^{3}$, Brad J. Schoenfeld ${ }^{4}$ and Zeljko Pedisic ${ }^{1}$
}

\begin{abstract}
Background: We aimed to perform an umbrella review of meta-analyses examining the effects of sodium bicarbonate supplementation on exercise performance.

Methods: We systematically searched for meta-analyses that examined the effects of sodium bicarbonate supplementation on exercise performance. The methodological quality of the included reviews was evaluated using the Assessing the Methodological Quality of Systematic Reviews 2 (AMSTAR 2) checklist. Grading of Recommendations Assessment, Development, and Evaluation (GRADE) framework for downgrading the certainty in evidence was used, which included assessments of risk of bias, inconsistency, indirectness, imprecision, and publication bias.

Results: Eight reviews of moderate and high methodological quality met inclusion criteria. Using the GRADE framework, evidence for the ergogenic effects of sodium bicarbonate supplementation on peak and mean power in the Wingate test and Yo-Yo test performance was classified as being of moderate quality. The evidence for these outcomes did not receive a point on the indirectness GRADE item, as "serious indirectness" was detected. Lowquality evidence was found for the ergogenic effect of sodium bicarbonate supplementation on endurance events lasting $\sim 45 \mathrm{~s}$ to $8 \mathrm{~min}$, muscle endurance, and 2000-m rowing performance. Evidence for these outcomes was classified as low quality, given that risk of bias, indirectness, and publication bias were assessed as "unclear", "serious", and "strongly suspected", respectively. The ergogenic effects ranged from trivial (pooled effect size: 0.09) to large (pooled effect size: 1.26). Still, for most outcomes, sodium bicarbonate elicited comparable ergogenic effects. For example, sodium bicarbonate produced similar effects on performance in endurance events lasting $\sim 45$ $\mathrm{s}$ to $8 \mathrm{~min}$, muscle endurance tests, and Yo-Yo test (pooled effect size range: 0.36 to 0.40 ). No significant differences between the effects of sodium bicarbonate and placebo were found for general mean power, muscle strength, and repeated-sprint ability.
\end{abstract}

Conclusion: Based on meta-analyses of moderate to high quality, it can be concluded that sodium bicarbonate supplementation acutely enhances peak anaerobic power, anaerobic capacity, performance in endurance events lasting $\sim 45 \mathrm{~s}$ to $8 \mathrm{~min}$, muscle endurance, 2000-m rowing performance, and high-intensity intermittent running. More research is needed among women to improve the generalizability of findings.

\footnotetext{
* Correspondence: jozo.grgic@live.vu.edu.au

${ }^{1}$ Institute for Health and Sport, Victoria University, Melbourne, Australia

Full list of author information is available at the end of the article
}

(c) The Author(s). 2021 Open Access This article is licensed under a Creative Commons Attribution 4.0 International License, which permits use, sharing, adaptation, distribution and reproduction in any medium or format, as long as you give appropriate credit to the original author(s) and the source, provide a link to the Creative Commons licence, and indicate if changes were made. The images or other third party material in this article are included in the article's Creative Commons licence, unless indicated otherwise in a credit line to the material. If material is not included in the article's Creative Commons licence and your intended use is not permitted by statutory regulation or exceeds the permitted use, you will need to obtain permission directly from the copyright holder. To view a copy of this licence, visit http://creativecommons.org/licenses/by/4.0/. The Creative Commons Public Domain Dedication waiver (http://creativecommons.org/publicdomain/zero/1.0/) applies to the data made available in this article, unless otherwise stated in a credit line to the data. 


\section{Background}

Sodium bicarbonate is a popular nutritional supplement, with studies exploring its effects on exercise performance dating back to the 1930s [1]. The effects of sodium bicarbonate supplementation have been investigated for different exercise tasks, varying in duration and intensity (e.g., high-intensity running or cycling, 200-m swimming, boxing, resistance exercise, 2000-m rowing, and repeated-sprint performance) [2-11]. However, the findings have been inconsistent, with studies reporting ergogenic, ergolytic and no significant effects [2-11]. Some of the inconsistencies between findings may be due to differences in the population analyzed, sodium bicarbonate supplementation protocols, exercise protocol, and performance outcomes. Besides these factors, the discrepancies in the findings might be due to the small sample sizes in some of the individual studies, which might have resulted in low statistical power [12]. Specifically, several studies published on this topic were performed while including only 5 to 6 participants [7-10]. One way to overcome the issue with small sample sizes in primary studies is to pool their results in a metaanalysis.

In recent years, several research groups performed meta-analyses examining the effects of sodium bicarbonate supplementation on different aspects of exercise performance [13-20]. However, meta-analyses tend to be narrow in scope. Specifically, they commonly concentrate on one specific outcome or a particular population [21]. Due to this limitation, it may be challenging to establish conclusive recommendations regarding the overall effect of sodium bicarbonate supplementation on exercise performance.

Given the increased popularity of meta-analyses, researchers have recently started to perform umbrella reviews, which endeavor to synthesize and critically evaluate information from all meta-analyses performed on a given topic $[21,22]$. As several meta-analyses [1320] explored the effects of sodium bicarbonate supplementation on exercise performance, it is timely to summarize their findings in the form of an umbrella review. Such a review is needed to: (i) evaluate the overall efficacy of sodium bicarbonate supplementation in improving exercise performance, (ii) assess the availability and quality of meta-analytic evidence, and (iii) provide recommendations for future research. Therefore, the aim of this paper was to perform an umbrella review of meta-analyses exploring the effects of sodium bicarbonate supplementation on exercise performance.

\section{Methods}

\section{Search strategy}

The literature search was performed across the following five databases: CINAHL, PubMed/MEDLINE, Scopus,
SPORTDiscus and Web of Science. The search was performed on December 11th, 2020, using the following search syntax: ("sodium bicarbonate" OR NaHCO3) AND (exercise OR training OR muscle OR "physical performance" OR "aerobic endurance" OR "peak power" OR "mean power") AND (meta-an" OR "systematic review"). The search was performed through the titles, abstracts and keywords of documents indexed in the databases between the database inception and the search date. The search and selection of meta-analyses were performed independently by two authors (JG and IG). Upon completion, the lists of included and excluded reviews were compared. Of note, the list of included papers was the same between these two authors.

\section{Inclusion criteria}

The reviews that satisfied the following criteria were included in this umbrella review: (a) examined the effects of sodium bicarbonate supplementation on exercise performance in human participants, (b) analyzed the data using a meta-analysis and (c) published in English. The following criteria were outlined in the ParticipantIntervention-Comparison-Outcome (PICO) process:

- Participants: healthy individuals, not limited to sex or age.

- Interventions: sodium bicarbonate supplementation.

- Comparison group: placebo.

- Outcome measures: exercise performance.

\section{Data extraction}

Data extraction was performed following previous recommendations for umbrella reviews [23]. From each included review, we extracted the following data: (a) number of included studies, (b) pooled number of participants, (c) exercise test/outcome and (d) pooled effect sizes with their $95 \%$ confidence intervals (CI), $p$-values and $I^{2}$. Data extraction was performed in duplicate by two authors (JG and IG) of the review. Data extraction files were compared between the authors and all observed differences were scrutinized and corrected. For meta-analyses that used Cohen's $d$, the pooled effect size was classified as "small" (Cohen's $d:$ : 0.20-0.49), "medium" (Cohen's $d$ : 0.50-0.79), and "large" (Cohen's $d: \geq 0.80$ ), according to Cohen [24].

\section{Methodological quality}

We evaluated the methodological quality of the included reviews using the Assessing the Methodological Quality of Systematic Reviews 2 (AMSTAR 2) checklist [25]. We opted to use the AMSTAR 2 checklist because it is one of the most widely used instruments for the assessment of the quality of reviews and has also been previously applied in the field of sports nutrition [25, 26]. This 
checklist has a total of 16 items that include questions regarding the use of PICO, review registration, study inclusion criteria, comprehensiveness of the search strategy, number of authors that performed the search and data extraction, presentation of included and excluded studies, use of a scale for the evaluation of methodological quality, received funding (for authors of both primary studies and reviews), appropriateness of the meta-analysis model, mention and interpretation of heterogeneity between included studies, and investigation of publication bias. There are four possible answers in each item: "yes", "no", "cannot answer" and "not applicable". "Yes" is the only answer that earns a point on a given item. Therefore, the maximum score on the checklist is 16 . The quality of included reviews was classified as "low", "moderate" or "high", if less than $40 \%$ of items were satisfied, between 40 and $80 \%$ of items were satisfied and at least $80 \%$ of items were satisfied, respectively [26].

\section{Quality of evidence}

Grading of Recommendations Assessment, Development, and Evaluation (GRADE) framework for downgrading the certainty in evidence was used to evaluate the quality of evidence [27]. In brief, GRADE provides a systematic method for assessing the certainty of findings in meta-analyses, and thus affords the ability to draw conclusions on the strength of practical recommendations. We used a modified GRADE scale that evaluates the risk of bias, inconsistency, indirectness, imprecision, and publication bias. Specific details on the use of this scale are reported elsewhere [26, 27]. Based on the GRADE evaluation, the quality of evidence was classified as "very low", "low", "moderate" or "high". The methodological quality and quality of evidence were evaluated independently by two authors of this review (JG and IG). Upon completion, scores were compared between the authors and all observed differences were scrutinized and corrected.

\section{Results}

\section{Search results}

In the five databases explored, the search syntax yielded a total of 123 results. Out of this number of search results, 15 full-text papers were read [13-20, 28-34], while other references were excluded based on their titles and/ or abstracts. After reading the full-texts, seven reviews were excluded because they did not contain a metaanalysis [28-34]. Two of these reviews [31, 32] calculated effect sizes from the included primary studies but did not pool them using a meta-analysis, and, thus, they did not satisfy the inclusion criteria. Therefore, a total of eight meta-analyses [13-20] were included in the current umbrella review (Fig. 1).

\section{Summary of the included reviews}

The number of studies included in each meta-analysis ranged from 5 to 26 (average: 13 studies; Table 1). The pooled number of participants per meta-analysis ranged from 46 to 241 . The majority of participants in primary studies were males (77 to $100 \%$ of all participants included in the meta-analyses). The meta-analyses explored the effects of sodium bicarbonate supplementation on a range of exercise outcomes, including: general mean power (calculated as the change in performance in cycling, running, rowing or swimming tasks in highintensity trials of short duration; 26 included studies), performance in endurance events lasting $\sim 4 \mathrm{~s}$ to $8 \mathrm{~min}$ (operationally defined as the time needed to complete an event of swimming, running, cycling or rowing; 25 included studies), muscle endurance (12 included studies), muscle strength (11 included studies), peak and mean power in single and repeated Wingate tests $(3,6$, or 10 included studies), repeated-sprint performance (total work, best sprint and last sprint performance; 6 included studies), and Yo-Yo test performance (5 included studies).

\section{Methodological quality and quality of evidence}

The average score on the AMSTAR 2 checklist was $70 \%$ (range: 50 to $81 \%$ ). Six reviews were categorized as being of moderate methodological quality, while two reviews were categorized as being of high quality (Table 2). Based on the GRADE checklist, the quality of evidence ranged from low to moderate. Low-quality evidence was found for general mean power, performance in endurance events lasting $\sim 45 \mathrm{~s}$ to $8 \mathrm{~min}$, muscle endurance, mean and peak power output in the Wingate test (in one of two meta-analyses that explored this outcome), total work in repeated-sprints, and 2000-m rowing performance. Moderate quality evidence was found for muscle strength, peak and mean power in the Wingate test, best sprint and last sprint performance in repeatedsprints, and Yo-Yo test performance (Table 3).

\section{Effects of sodium bicarbonate supplementation on exercise performance Meta-analyses that used Cohen's $d$}

A meta-analysis of 25 studies reported ergogenic effects of sodium bicarbonate supplementation on performance in endurance events lasting $\sim 45 \mathrm{~s}$ to $8 \mathrm{~min}$ (pooled effect size: 0.40; Fig. 2; Table 4) [14].

In a meta-analysis including data from 12 studies, sodium bicarbonate supplementation was found to be ergogenic for muscular endurance (pooled effect size: 0.37) [17]. In a meta-analysis including 11 studies, no 


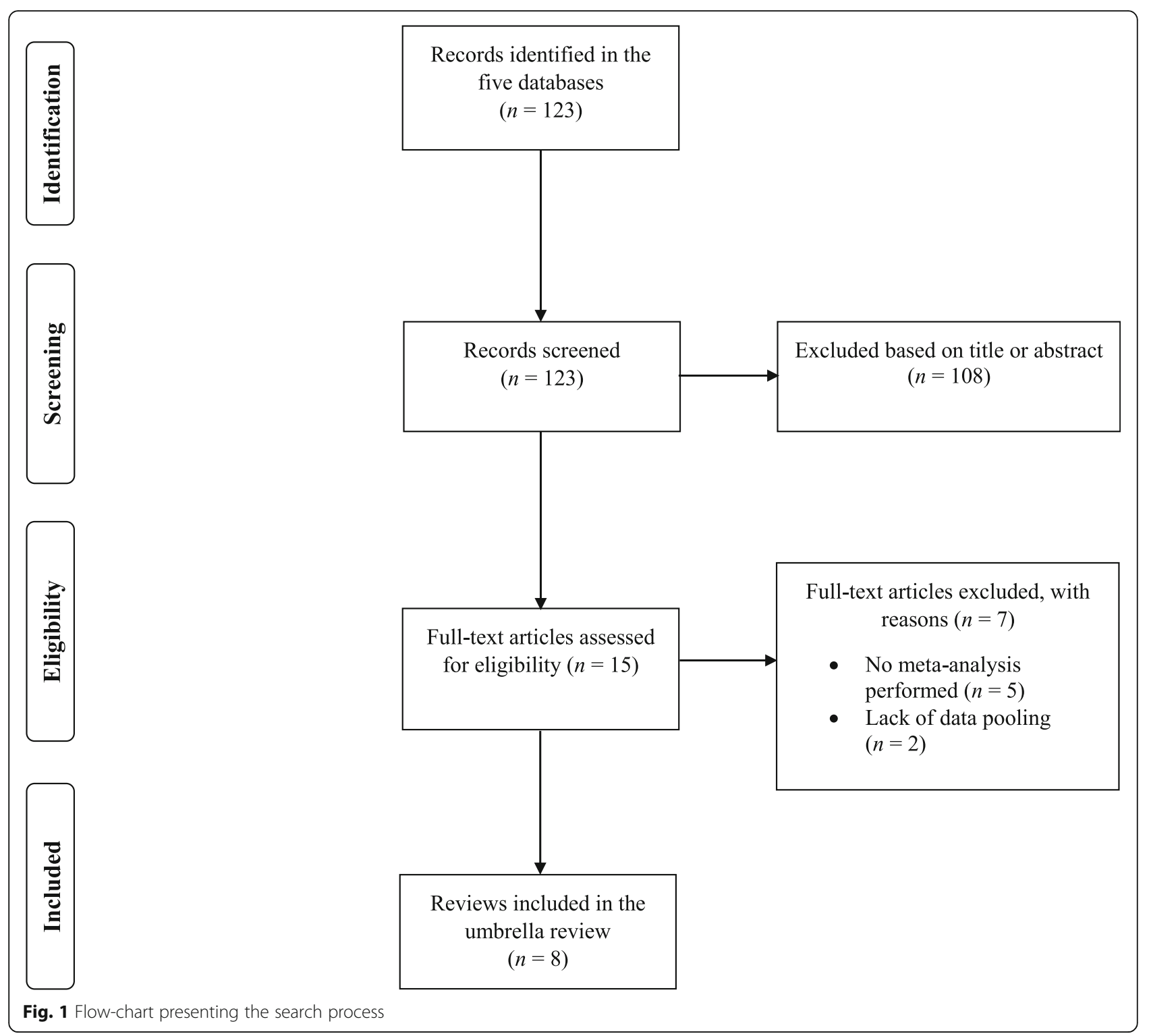

ergogenic effect was observed for muscular strength (pooled effect size: - 0.03) [17].

Two meta-analyses examined the effects of acute sodium bicarbonate ingestion on mean and peak power in the single and repeated Wingate tests $[15,19]$. In a meta-analysis that included six studies, there were no ergogenic effects of acute sodium bicarbonate ingestion on mean and peak power in Wingate bout 1,2 and 3 (pooled effect size range: -0.07 to 0.22) [19]. In another meta-analysis with 10 included studies [15], an ergogenic effect of sodium bicarbonate supplementation was found on mean power in Wingate bout 2 (pooled effect size: 0.09 ) and bout 4 (pooled effect size: 0.62 ), as well as peak power in Wingate bout 3 (pooled effect size: 0.09). No significant differences between the effects of sodium bicarbonate and placebo were observed in other comparisons (i.e., mean power in Wingate bout 1 and 3 and peak power in Wingate bout 1,2 and 4).

The effects of multi-day protocols of sodium bicarbonate ingestion on mean and peak power in single and repeated Wingate tests were examined in one metaanalysis that included 3 studies [19]. Sodium bicarbonate ingestion was ergogenic for peak and mean power (pooled effect size range: 1.21 to 1.26).

The effects of sodium bicarbonate supplementation on repeated-sprint performance measures were explored in one meta-analysis that included 6 studies [18]. No significant difference between the effects of sodium bicarbonate and placebo was found for any of the analyzed outcomes (pooled effect size range: 0.02 to 0.43 ). 
Table 1 Summary of the meta-analyses included in the umbrella review

\begin{tabular}{|c|c|c|c|c|c|}
\hline Reference & Included studies & $\begin{array}{l}\text { Number of included primary } \\
\text { studies (sample size) }\end{array}$ & Performance test/outcome & $\begin{array}{l}\text { Effect size } \\
\text { and } p \text {-value }\end{array}$ & $r^{2}$ \\
\hline \multicolumn{6}{|c|}{ Meta-analyses that used Cohen's $d$ for data analysis } \\
\hline $\begin{array}{l}\text { Christensen } \\
\text { et al. [14] }\end{array}$ & $\begin{array}{l}\text { Crossover study } \\
\text { designs }\end{array}$ & 25 studies $(n=235)$ & $\begin{array}{l}\text { Endurance events lasting } \sim 45 \mathrm{~s} \text { to } \\
8 \mathrm{~min}\end{array}$ & $\begin{array}{l}0.40 \text { (95\% Cl: } 0.27,0.54) ; \\
p<0.001\end{array}$ & $\mathrm{n} / \mathrm{a}$ \\
\hline Grgic [15] & $\begin{array}{l}\text { Crossover study } \\
\text { designs }\end{array}$ & 10 studies $(n=102)$ & $\begin{array}{l}\text { Mean and peak power in single } \\
\text { and repeated Wingate tests }\end{array}$ & 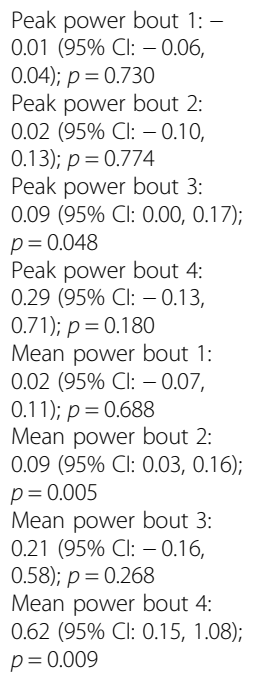 & $\begin{array}{l}\text { Peak power } \\
\text { bout 1: } 0 \% \\
\text { Peak power } \\
\text { bout 2: } 7 \% \\
\text { Peak power } \\
\text { bout 3: } 0 \% \\
\text { Peak power } \\
\text { bout 4: } 0 \% \\
\text { Mean power } \\
\text { bout 1: } 0 \% \\
\text { Mean power } \\
\text { bout 2: } 0 \% \\
\text { Mean power } \\
\text { bout 3: } 0 \% \\
\text { Mean power } \\
\text { bout 4: } 0 \%\end{array}$ \\
\hline $\begin{array}{l}\text { Grgic et al. } \\
{[16]}\end{array}$ & $\begin{array}{l}\text { Crossover study } \\
\text { designs }\end{array}$ & 5 studies $(n=46)$ & Yo-Yo test performance & $\begin{array}{l}0.36(95 \% \text { Cl: } 0.10,0.63) \\
p=0.007\end{array}$ & $14 \%$ \\
\hline $\begin{array}{l}\text { Grgic et al. } \\
{[17]}\end{array}$ & $\begin{array}{l}\text { Crossover study } \\
\text { designs }\end{array}$ & $\begin{array}{l}13 \text { studies for muscle endurance }(n=113)^{\mathrm{a}} \text { and } 11 \\
\text { studies for muscle strength }(n=110)\end{array}$ & $\begin{array}{l}\text { Muscle endurance and muscle } \\
\text { strength }\end{array}$ & $\begin{array}{l}\text { Muscle endurance: } 0.37 \\
(95 \% \text { Cl: } 0.15,0.59) ; p= \\
0.001 \\
\text { Muscle strength: }-0.03 \\
(95 \% \text { Cl: }-0.18,0.12) ; \\
p=0.725\end{array}$ & $\begin{array}{l}\text { Muscle } \\
\text { endurance: } \\
70 \% \\
\text { Muscle } \\
\text { strength: } \\
45 \%\end{array}$ \\
\hline $\begin{array}{l}\text { Lopes-Silva } \\
\text { et al. [18] }\end{array}$ & $\begin{array}{l}\text { Crossover study } \\
\text { designs }\end{array}$ & 6 studies $(n=77)$ & $\begin{array}{l}\text { Measures of repeated-sprint ability } \\
\text { (total work, best sprint, and last } \\
\text { sprint performance) }\end{array}$ & $\begin{array}{l}\text { Total work: } 0.43(95 \% \\
\text { Cl: }-0.11,0.97) ; p=0.12 \\
\text { Best sprint: } 0.02(95 \% \\
\text { Cl: }-0.30,0.34) ; p=0.90 \\
\text { Last sprint: } 0.20(95 \% \\
\text { Cl: }-0.13,0.52) ; p=0.14\end{array}$ & $\begin{array}{l}\text { Total work: } \\
\text { 0\% } \\
\text { Best sprint: } \\
\text { 0\% } \\
\text { Last sprint: } \\
69 \%\end{array}$ \\
\hline $\begin{array}{l}\text { Lopes-Silva } \\
\text { et al. [19] }\end{array}$ & $\begin{array}{l}\text { Crossover and } \\
\text { between-group } \\
\text { study designs }\end{array}$ & $\begin{array}{l}6 \text { studies }(n=65) \text { that used protocols of acute } \\
\text { ingestion and } 3 \text { studies }(n=60) \text { that used multi-day } \\
\text { protocols of ingestion }\end{array}$ & $\begin{array}{l}\text { Mean and peak power in single } \\
\text { and repeated Wingate tests }\end{array}$ & $\begin{array}{l}\text { Acute ingestion } \\
\text { Peak power: } 0.02(95 \% \\
\text { Cl: }-0.19,0.23) ; p=0.87 \\
\text { Mean power: } 0.15(95 \% \\
\text { Cl: }-0.06,0.36) ; p=0.92 \\
\text { Multi-day protocols of } \\
\text { ingestion } \\
\text { Peak power: } 1.21(95 \% \\
\text { Cl: } 0.83,1.42) ; p=0.001 \\
\text { Mean power: } 1.26(95 \% \\
\text { Cl: } 0.96,1.56) ; p=0.001\end{array}$ & $\begin{array}{l}\text { Acute } \\
\text { ingestion } \\
\text { Peak power: } \\
0 \% \\
\text { Mean power: } \\
0 \% \\
\text { Multi-day } \\
\text { protocols of } \\
\text { ingestion } \\
\text { Peak power: } \\
27 \% \\
\text { Mean power: } \\
88 \%\end{array}$ \\
\hline \multicolumn{6}{|c|}{ Meta-analyses that used percent changes for data analysis } \\
\hline $\begin{array}{l}\text { Carr et al. } \\
{[13]}\end{array}$ & $\begin{array}{l}\text { Crossover study } \\
\text { designs }\end{array}$ & 26 studies $(n=241)$ & General mean power & $\begin{array}{l}1.7 \%(90 \% \mathrm{CL}: 90 \% \mathrm{CL}: \\
-0.3 \%, 3.7)\end{array}$ & $\mathrm{n} / \mathrm{a}$ \\
\hline $\begin{array}{l}\text { Turnes et al. } \\
{[20]}\end{array}$ & $\begin{array}{l}\text { Crossover study } \\
\text { designs }\end{array}$ & 5 studies $(n=52)$ & Mean power in 2000-m rowing & $\begin{array}{l}1.4 \%(90 \% \mathrm{CL}: 0.1 \\
2.6 \%)\end{array}$ & $n / a$ \\
\hline
\end{tabular}

Cl: confidence interval; CL: confidence limit; ${ }^{\mathrm{a}} 12$ studies were included in the meta-analysis

One meta-analysis, including 5 studies, examined the effects of sodium bicarbonate supplementation on YoYo test performance and reported an ergogenic effect of sodium bicarbonate (pooled effect size: 0.36 ) [16].

\section{Meta-analyses that used percent changes}

In one meta-analysis [13], exercise performance data from 25 included studies were converted to general mean power. In this analysis, there was no significant 
Table 2 Result of the quality assessment using the Assessing the Methodological Quality of Systematic Reviews 2 (AMSTAR 2) checklist

\begin{tabular}{|c|c|c|c|c|c|c|c|c|c|c|c|c|c|c|c|c|c|}
\hline \multirow[t]{2}{*}{ Reference } & \multicolumn{16}{|c|}{ AMSTAR 2 items } & \multirow[t]{2}{*}{ Score } \\
\hline & 1 & 2 & 3 & 4 & 5 & 6 & 7 & 8 & 9 & 10 & 11 & 12 & 13 & 14 & 15 & $\overline{16}$ & \\
\hline Carr et al. [13] & Yes & No & Yes & Yes & Yes & Yes & No & Yes & No & No & Yes & $\mathrm{n} / \mathrm{a}$ & $\mathrm{n} / \mathrm{a}$ & Yes & Yes & Yes & $\begin{array}{l}63 \% \\
M Q\end{array}$ \\
\hline Christensen et al. [14] & Yes & No & Yes & Yes & Yes & Unclear & No & Yes & Yes & Yes & Yes & Yes & Yes & Yes & No & Yes & $\begin{array}{l}75 \% \\
M Q\end{array}$ \\
\hline Grgic [15] & Yes & No & Yes & Yes & No & No & No & Yes & Yes & No & Yes & Yes & Yes & Yes & Yes & Yes & $\begin{array}{l}69 \% \\
M Q\end{array}$ \\
\hline Grgic et al. [16] & Yes & No & Yes & Yes & Yes & Yes & No & Yes & Yes & No & Yes & Yes & Yes & Yes & Yes & Yes & $\begin{array}{l}81 \% \\
\mathrm{HQ}\end{array}$ \\
\hline Grgic et al. [17] & Yes & No & Yes & Yes & Yes & Yes & No & Yes & Yes & No & Yes & Yes & Yes & Yes & Yes & Yes & $\begin{array}{l}81 \% \\
\mathrm{HQ}\end{array}$ \\
\hline Lopes-Silva et al. [18] & Yes & No & Yes & Yes & Yes & Unclear & No & Yes & Yes & No & Yes & Yes & Yes & Yes & Yes & Yes & $\begin{array}{l}75 \% \\
M Q\end{array}$ \\
\hline Lopes-Silva et al. [19] & Yes & No & Yes & Yes & Yes & Unclear & No & Yes & Yes & No & Yes & Yes & Yes & Yes & No & Yes & $\begin{array}{l}69 \% \\
M Q\end{array}$ \\
\hline Turnes et al. [20] & Yes & No & Yes & Yes & Unclear & Unclear & No & Yes & No & No & Yes & $\mathrm{n} / \mathrm{a}$ & $\mathrm{n} / \mathrm{a}$ & Yes & Yes & Yes & $\begin{array}{l}50 \% \\
\mathrm{MQ}\end{array}$ \\
\hline
\end{tabular}

MQ: moderate quality; $\mathrm{HQ}$ : high quality

difference between sodium bicarbonate and placebo (1.7\%; 90\% confidence limit [CL]: - 0.3, 3.7\%; Fig. 3).

One meta-analysis included 6 studies on the effects of sodium bicarbonate supplementation on 2000-m rowing performance [20]. Sodium bicarbonate supplementation was found to enhance this outcome by $1.4 \%$ (90\% CL: $0.1,2.6 \%)$.

\section{Subgroup analyses}

Besides the main analyses, three reviews $[13,17,19]$ also conducted additional subgroup meta-analyses (Table 5). In the meta-analysis [13] with general mean power as the outcome variable, subgroup analyses included sodium bicarbonate dose, number of exercise bouts, exercise test duration, participants' sex and training status, and non-blinded (blinding of participants) study designs. A significant ergogenic effect of sodium bicarbonate (0.6\%; 90\% CL: $0.2,1.0 \%)$ was found only when five extra exercise bouts were performed.

In the review with muscle endurance and muscle strength as outcome variables [17], subgroup metaanalyses included the size of the exercised muscle, protocol of ingestion, and testing in a fatigued vs. nonfatigued state. The results of these subgroup metaanalyses were consistent with those reported in the primary meta-analyses, confirming an ergogenic effect of sodium bicarbonate on muscle endurance and finding no significant difference between the effects of sodium bicarbonate and placebo on muscle strength (Table 5).

Finally, Lopes-Silva et al. [19] performed subgroup analyses to explore the effects of single-dose and multi- day protocols of sodium bicarbonate ingestion on peak and mean power in Wingate bouts $1-4$. As with the primary findings, subgroup analyses found that multi-day protocol of supplementation increased peak and mean power in bouts 1-4. No significant difference was found between the effects of placebo and acute sodium bicarbonate ingestion.

\section{Discussion}

\section{Main findings of the review}

Based on the meta-analytic evidence, it can be concluded that sodium bicarbonate supplementation acutely enhances peak anaerobic power, anaerobic capacity, performance in endurance events lasting $\sim 45 \mathrm{~s}$ to $8 \mathrm{~min}$, muscle endurance, 2000-m rowing performance, and high-intensity intermittent running. This conclusion is based on reviews of moderate and high methodological quality. Moderate quality evidence was found for the ergogenic effects of sodium bicarbonate supplementation on peak and mean power in the Wingate test and Yo-Yo test performance. Low quality evidence was found for the ergogenic effect of sodium bicarbonate supplementation on performance in endurance events lasting $\sim 45 \mathrm{~s}$ to $8 \mathrm{~min}$, muscle endurance, and 2000-rowing performance. The ergogenic effects ranged from trivial (pooled effect size: 0.09 ) to large (pooled effect size: 1.26). Still, for most outcomes, sodium bicarbonate elicited comparable ergogenic effects. For example, sodium bicarbonate produced similar effects on performance in endurance events lasting $\sim 45 \mathrm{~s}$ to $8 \mathrm{~min}$, muscle endurance tests, and Yo-Yo test (pooled effect size range: 0.36 to 0.40 ). 
Table 3 Results of the quality of evidence assessment using the Grading of Recommendations Assessment, Development, and Evaluation (GRADE) criteria

\begin{tabular}{|c|c|c|c|c|c|c|}
\hline \multirow[t]{2}{*}{ Reference } & \multicolumn{5}{|l|}{ GRADE items } & \multirow{2}{*}{$\begin{array}{l}\text { Quality of } \\
\text { the } \\
\text { evidence* }\end{array}$} \\
\hline & Risk of bias & Inconsistency & Indirectness & Imprecision & Publication bias & \\
\hline $\begin{array}{l}\text { Carr et al. } \\
{[13]}\end{array}$ & $\begin{array}{l}\text { Unclear (no } \\
\text { quality } \\
\text { assessment } \\
\text { performed) }\end{array}$ & Not serious & $\begin{array}{l}\text { Serious indirectness (only 15\% of } \\
\text { participants included in the } \\
\text { analysis were women) }\end{array}$ & Not serious & Undetected & $\begin{array}{l}\text { Low } \\
\oplus \oplus \bigcirc \mathrm{O}\end{array}$ \\
\hline $\begin{array}{l}\text { Christensen } \\
\text { et al. [14] }\end{array}$ & Not serious & Not serious & $\begin{array}{l}\text { Serious indirectness (only 9\% of } \\
\text { participants included in the } \\
\text { analysis were women) }\end{array}$ & Not serious & $\begin{array}{l}\text { Strongly suspected (no "grey" literature } \\
\text { searches; asymmetry of the funnel plot } \\
\text { was not explored; the effect size of the } \\
\text { largest study was smaller than the pooled } \\
\text { estimate) }\end{array}$ & $\begin{array}{l}\text { Low } \\
\oplus \oplus \bigcirc O\end{array}$ \\
\hline \multirow[t]{2}{*}{ Grgic [15] } & $\begin{array}{l}\text { Peak power: } \\
\text { not serious }\end{array}$ & $\begin{array}{l}\text { Peak power: } \\
\text { not serious }\end{array}$ & $\begin{array}{l}\text { Peak power: serious indirectness } \\
\text { (none of the participants } \\
\text { included in the analysis were } \\
\text { women) }\end{array}$ & $\begin{array}{l}\text { Peak power: } \\
\text { not serious }\end{array}$ & Peak power: undetected & $\begin{array}{l}\text { Peak } \\
\text { power: } \\
\text { moderate } \\
\oplus \oplus \oplus 0\end{array}$ \\
\hline & $\begin{array}{l}\text { Mean } \\
\text { power: not } \\
\text { serious }\end{array}$ & $\begin{array}{l}\text { Mean power: } \\
\text { not serious }\end{array}$ & $\begin{array}{l}\text { Mean power: serious indirectness } \\
\text { (only } 15 \% \text { of participants } \\
\text { included in the analysis were } \\
\text { women) }\end{array}$ & $\begin{array}{l}\text { Mean } \\
\text { power: not } \\
\text { serious }\end{array}$ & Mean power: undetected & $\begin{array}{l}\text { Mean } \\
\text { power: } \\
\text { moderate } \\
\oplus \oplus \oplus 0\end{array}$ \\
\hline $\begin{array}{l}\text { Grgic et al. } \\
{[16]}\end{array}$ & Not serious & Not serious & $\begin{array}{l}\text { Serious indirectness (none of the } \\
\text { participants included in the } \\
\text { analysis were women) }\end{array}$ & Not serious & Undetected & $\begin{array}{l}\text { Moderate } \\
\oplus \oplus \oplus O\end{array}$ \\
\hline \multirow[t]{2}{*}{$\begin{array}{l}\text { Grgic et al. } \\
{[17]}\end{array}$} & $\begin{array}{l}\text { Muscle } \\
\text { endurance: } \\
\text { not serious }\end{array}$ & Not serious & $\begin{array}{l}\text { Serious indirectness (only 5\% of } \\
\text { participants included in the } \\
\text { analysis were women) }\end{array}$ & Not serious & $\begin{array}{l}\text { Strongly suspected ("grey" literature } \\
\text { searches were performed; however, } \\
\text { asymmetry of the funnel plot was not } \\
\text { explored and the effect size of the largest } \\
\text { study was smaller than the pooled } \\
\text { estimate) }\end{array}$ & $\begin{array}{l}\text { Low } \\
\oplus \oplus \bigcirc \bigcirc\end{array}$ \\
\hline & $\begin{array}{l}\text { Muscle } \\
\text { strength: } \\
\text { not serious }\end{array}$ & Not serious & $\begin{array}{l}\text { Serious indirectness (only } 4 \% \text { of } \\
\text { participants included in the } \\
\text { analysis were women) }\end{array}$ & Not serious & Undetected & $\begin{array}{l}\text { Moderate } \\
\oplus \oplus \oplus \mathrm{O}\end{array}$ \\
\hline \multirow[t]{3}{*}{$\begin{array}{l}\text { Lopes-Silva } \\
\text { et al. [18] }\end{array}$} & $\begin{array}{l}\text { Total work: } \\
\text { not serious }\end{array}$ & $\begin{array}{l}\text { Total work: } \\
\text { not serious }\end{array}$ & $\begin{array}{l}\text { Total work: serious indirectness } \\
\text { (only } 28 \% \text { of participants } \\
\text { included in the analysis were } \\
\text { women) }\end{array}$ & $\begin{array}{l}\text { Total work: } \\
\text { serious } \\
\text { limitation }\end{array}$ & Total work: undetected & $\begin{array}{l}\text { Low } \\
\oplus \oplus \bigcirc \mathrm{O}\end{array}$ \\
\hline & $\begin{array}{l}\text { Best sprint: } \\
\text { not serious }\end{array}$ & $\begin{array}{l}\text { Best sprint: } \\
\text { not serious }\end{array}$ & $\begin{array}{l}\text { Best sprint: serious indirectness } \\
\text { (only } 23 \% \text { of participants } \\
\text { included in the analysis were } \\
\text { women) }\end{array}$ & $\begin{array}{l}\text { Best sprint: } \\
\text { not serious }\end{array}$ & Best sprint: undetected & $\begin{array}{l}\text { Moderate } \\
\oplus \oplus \oplus \mathrm{O}\end{array}$ \\
\hline & $\begin{array}{l}\text { Last sprint: } \\
\text { not serious }\end{array}$ & $\begin{array}{l}\text { Last sprint: } \\
\text { not serious }\end{array}$ & $\begin{array}{l}\text { Last sprint: serious indirectness } \\
\text { (only } 23 \% \text { of participants } \\
\text { included in the analysis were } \\
\text { women) }\end{array}$ & $\begin{array}{l}\text { Last sprint: } \\
\text { not serious }\end{array}$ & Last sprint: undetected & $\begin{array}{l}\text { Moderate } \\
\oplus \oplus \oplus \mathrm{O}\end{array}$ \\
\hline \multirow[t]{4}{*}{$\begin{array}{l}\text { Lopes-Silva } \\
\text { et al. [19] }\end{array}$} & $\begin{array}{l}\text { Acute } \\
\text { ingestion, } \\
\text { peak power: } \\
\text { not serious }\end{array}$ & $\begin{array}{l}\text { Acute } \\
\text { ingestion, } \\
\text { peak power: } \\
\text { not serious }\end{array}$ & $\begin{array}{l}\text { Acute ingestion, peak power: } \\
\text { serious indirectness (none of the } \\
\text { participants included in the } \\
\text { analysis were women) }\end{array}$ & $\begin{array}{l}\text { Acute } \\
\text { ingestion, } \\
\text { peak power: } \\
\text { not serious }\end{array}$ & $\begin{array}{l}\text { Acute ingestion, peak power: strongly } \\
\text { suspected (no "grey" literature searches; } \\
\text { asymmetry of the funnel plot was not } \\
\text { explored; the effect size of the largest } \\
\text { study was similar to the pooled estimate) }\end{array}$ & $\begin{array}{l}\text { Low } \\
\oplus \oplus \mathrm{OO}\end{array}$ \\
\hline & $\begin{array}{l}\text { Acute } \\
\text { ingestion, } \\
\text { mean } \\
\text { power: not } \\
\text { serious }\end{array}$ & $\begin{array}{l}\text { Acute } \\
\text { ingestion, } \\
\text { mean power: } \\
\text { not serious }\end{array}$ & $\begin{array}{l}\text { Acute ingestion, mean power: } \\
\text { serious indirectness (none of the } \\
\text { participants included in the } \\
\text { analysis were women) }\end{array}$ & $\begin{array}{l}\text { Acute } \\
\text { ingestion, } \\
\text { mean } \\
\text { power: not } \\
\text { serious }\end{array}$ & $\begin{array}{l}\text { Acute ingestion, mean power: strongly } \\
\text { suspected (no "grey" literature searches; } \\
\text { asymmetry of the funnel plot was not } \\
\text { explored; the effect size of the largest } \\
\text { study was similar to the pooled estimate) }\end{array}$ & $\begin{array}{l}\text { Low } \\
\oplus \oplus \bigcirc \bigcirc\end{array}$ \\
\hline & $\begin{array}{l}\text { Multi-day } \\
\text { ingestion, } \\
\text { peak power: } \\
\text { not serious }\end{array}$ & $\begin{array}{l}\text { Multi-day } \\
\text { ingestion, } \\
\text { peak power: } \\
\text { not serious }\end{array}$ & $\begin{array}{l}\text { Multi-day ingestion, peak power: } \\
\text { serious indirectness (none of the } \\
\text { participants included in the } \\
\text { analysis were women) }\end{array}$ & $\begin{array}{l}\text { Multi-day } \\
\text { ingestion, } \\
\text { peak power: } \\
\text { not serious }\end{array}$ & $\begin{array}{l}\text { Multi-day ingestion, peak power: strongly } \\
\text { suspected (no "grey" literature searches; } \\
\text { asymmetry of the funnel plot was not } \\
\text { explored; the effect size of the largest } \\
\text { study was similar to the pooled estimate) }\end{array}$ & $\begin{array}{l}\text { Low } \\
\oplus \oplus \bigcirc \mathrm{O}\end{array}$ \\
\hline & $\begin{array}{l}\text { Multi-day } \\
\text { ingestion, }\end{array}$ & $\begin{array}{l}\text { Multi-day } \\
\text { ingestion, }\end{array}$ & $\begin{array}{l}\text { Multi-day ingestion, mean } \\
\text { power: serious indirectness }\end{array}$ & $\begin{array}{l}\text { Multi-day } \\
\text { ingestion, }\end{array}$ & $\begin{array}{l}\text { Multi-day ingestion, mean power: strongly } \\
\text { suspected (no "grey" literature searches; }\end{array}$ & $\begin{array}{l}\text { Low } \\
\oplus \oplus \bigcirc \bigcirc\end{array}$ \\
\hline
\end{tabular}


Table 3 Results of the quality of evidence assessment using the Grading of Recommendations Assessment, Development, and Evaluation (GRADE) criteria (Continued)

\begin{tabular}{|c|c|c|c|c|c|c|}
\hline \multirow[t]{2}{*}{ Reference } & \multicolumn{5}{|c|}{ GRADE items } & \multirow{2}{*}{$\begin{array}{l}\text { Quality of } \\
\text { the } \\
\text { evidence* }\end{array}$} \\
\hline & Risk of bias & Inconsistency & Indirectness & Imprecision & Publication bias & \\
\hline & $\begin{array}{l}\text { mean } \\
\text { power: not } \\
\text { serious }\end{array}$ & $\begin{array}{l}\text { mean power: } \\
\text { not serious }\end{array}$ & $\begin{array}{l}\text { (none of the participants } \\
\text { included in the analysis were } \\
\text { women) }\end{array}$ & $\begin{array}{l}\text { mean } \\
\text { power: not } \\
\text { serious }\end{array}$ & $\begin{array}{l}\text { asymmetry of the funnel plot was not } \\
\text { explored; the effect size of the largest } \\
\text { study was similar to the pooled estimate) }\end{array}$ & \\
\hline $\begin{array}{l}\text { Turnes et al. } \\
{[20]}\end{array}$ & $\begin{array}{l}\text { Unclear (no } \\
\text { quality } \\
\text { assessment } \\
\text { performed) }\end{array}$ & Not serious & $\begin{array}{l}\text { Serious indirectness (only } 10 \% \text { of } \\
\text { participants included in the } \\
\text { analysis were women) }\end{array}$ & Not serious & Undetected & $\begin{array}{l}\text { Low } \\
\oplus \oplus \mathrm{OO}\end{array}$ \\
\hline
\end{tabular}

Studies were classified as: $\oplus \oplus \oplus \oplus=$ high quality; $\oplus \oplus \oplus \mathrm{O}=$ moderate quality; $\oplus \oplus \mathrm{OO}=$ low quality; $\oplus \mathrm{OOO}=$ very low quality

No significant difference between the effects of sodium bicarbonate and placebo was found for general mean power, muscle strength and repeated-sprint ability.

\section{Generalizability of the results}

Most primary studies included in the meta-analyses were conducted among male participants, which limits the generalizability of findings. Specifically, 77 to $100 \%$ of participants included in the meta-analyses were males. Due to the uneven distribution of sexes in primary studies, all included reviews were categorized as having "serious indirectness" in the GRADE assessment [27]. One included review performed a subgroup analysis that only considered findings among females (pooled sample size $n=36$ ) [13]. In this subgroup analysis, the pooled effect of sodium bicarbonate supplementation on general mean power was $-0.7 \%$ (90\% confidence limit: $-2.1,0.7 \%$ ) and was concluded to be "unclear". However, it should be mentioned that no significant difference between sodium bicarbonate and placebo for general mean power was found in the main meta-analysis of this review (that included both females and males). While a handful of studies [35-38] conducted in females reported ergogenic effects of sodium bicarbonate on exercise performance, additional research in this population is needed to draw stronger inferences. Future studies should consider including both men and women and analyze their data separately to determine if there is a difference in responses to sodium bicarbonate supplementation between sexes. It should be noted, however, that such comparisons would require larger sample sizes to ensure adequate statistical power.

\section{Effects of sodium bicarbonate supplementation on exercise performance}

When ergogenic, it seems that the effectiveness of sodium bicarbonate supplementation is similar for different exercise tasks. Sodium bicarbonate was comparably ergogenic for performance in endurance events lasting $\sim 45 \mathrm{~s}$ to $8 \mathrm{~min}$, muscle endurance, and Yo-Yo test performance (pooled effect size range: 0.36 to 0.40 ) [14, $16,17]$. Therefore, it seems that small-to-moderate effects of sodium bicarbonate supplementation on exercise performance may be expected, which may be practically meaningful in sports training and competition. While this was not the main topic of this review, a brief mention of the mechanisms that explain these ergogenic effects is also needed. During intense exercise, the accumulation of $\mathrm{H}^{+}$and decrease in $\mathrm{pH}$ may contribute to fatigue and decreased performance due to its effects on glycolytic enzymes, $\mathrm{Ca}^{2+}$ sensitivity, and cross-bridge cycling $[39,40]$. Sodium bicarbonate ingestion may help to delay exercise-induced fatigue and improve performance, as it acts by increasing $\mathrm{H}^{+}$efflux and improving intramuscular acid-base (for a detailed review on the mechanisms, see [41]).

Most primary studies on this topic employed acute sodium bicarbonate supplementation protocols (e.g., a single dose consumed $3 \mathrm{~h}$ before exercise). However, one meta-analysis reported large effects among studies that used multi-day protocols of sodium bicarbonate supplementation [19]. Specifically, this meta-analysis included studies that provided daily sodium bicarbonate supplementation for 5 to 7 days before the exercise test (e.g., $4 \times 125 \mathrm{mg}$ per day), with additional sodium bicarbonate ingestion a few hours before the test. The advantage of this protocol is that it may reduce sodium bicarbonateinduced side effects, given that smaller doses are ingested throughout the day [41]. In this analysis, the effect size of sodium bicarbonate supplementation on peak and mean power recorded during single and repeated Wingate tests ranged from 0.79 to 2.03 . The effects of acute sodium bicarbonate supplementation (i.e., only a single dose consumed 90 to $180 \mathrm{~min}$ before exercise) on Wingate test performance were smaller (pooled effect size range: 0.09 to 0.62 ). This would suggest that greater ergogenic effects may be observed when using multi-day protocols of sodium bicarbonate supplementation. However, these findings were based on only three studies [42-44], none of which directly compared the utilized 


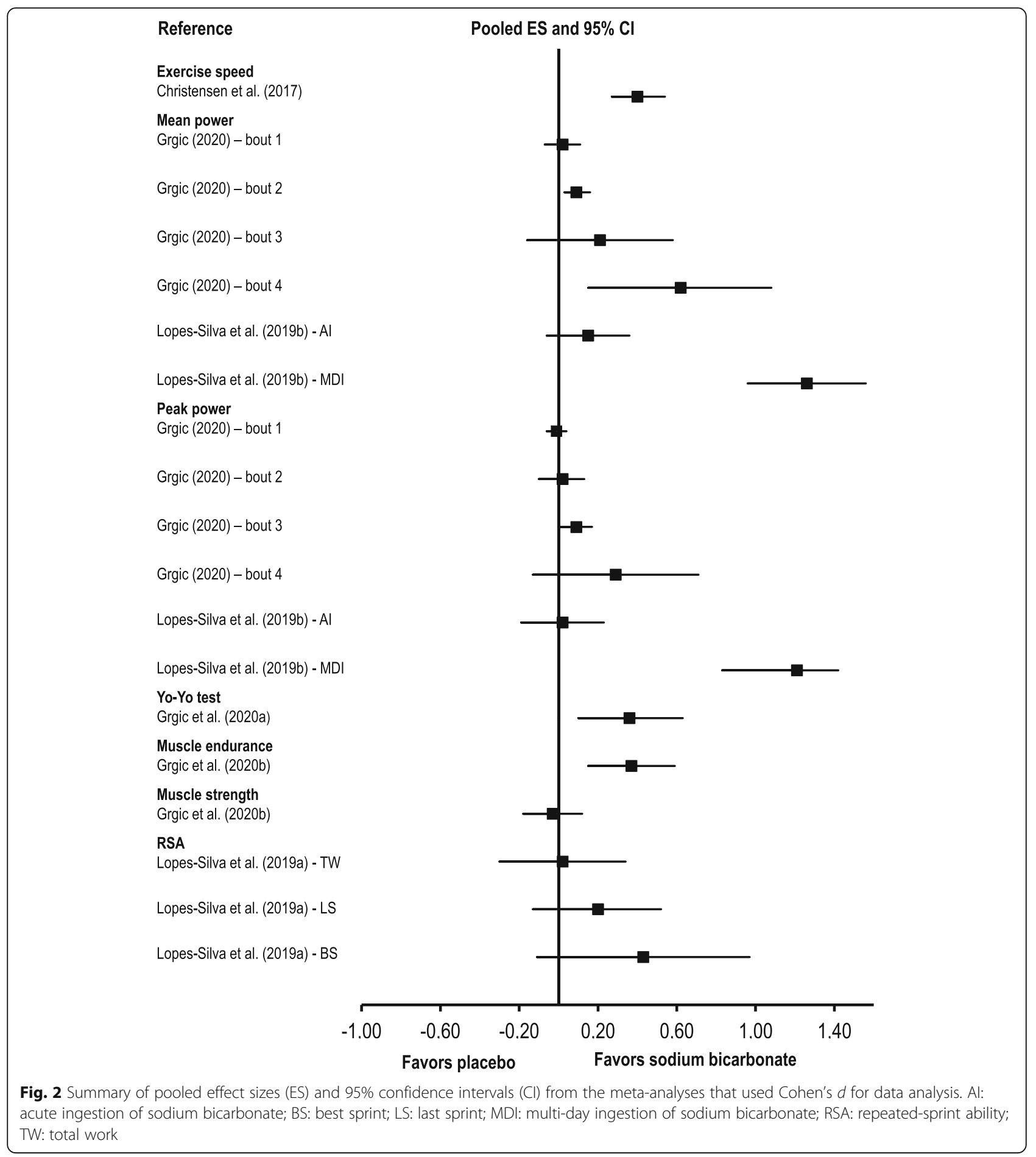

protocols of supplementation to protocols of acute sodium bicarbonate ingestion. Therefore, further research is needed to compare the effects of acute vs. multi-day protocols of sodium bicarbonate supplementation on different exercise tasks and in different populations. One study [45] explored the effects of both protocols and reported that they have similar ergogenic effects on repeated-sprint ability. Another study [46] compared the effects of single-dose and multi-day protocols of sodium bicarbonate ingestion on cycling performance on three consecutive testing days. While there was no significant difference between the protocols on the first testing day, 
Table 4 Effects of sodium bicarbonate supplementation on exercise performance: summary findings, methodological quality of literature reviews, and quality of evidence

\begin{tabular}{|c|c|c|}
\hline \multirow{2}{*}{$\begin{array}{l}\text { Quality of } \\
\text { evidence }\end{array}$} & \multicolumn{2}{|l|}{ Methodological quality of literature reviews } \\
\hline & Moderate & High \\
\hline \multicolumn{3}{|c|}{ Meta-analyses that found significant ergogenic effects of sodium bicarbonate } \\
\hline Low & $\begin{array}{l}\text { - Endurance events lasting } \sim 45 \text { s to } 8 \text { min in Christensen et al. [14] } \\
\text { - Anaerobic power in the Wingate test using the multiple-day supplementation protocol in } \\
\text { Lopes-Silva et al. [19] } \\
\text {-2000-m rowing in Turnes et al. [20] }\end{array}$ & $\begin{array}{l}\text { - Muscle endurance in Grgic et al. } \\
\text { [17] }\end{array}$ \\
\hline Moderate & - Anaerobic power in the repeated-bout Wingate test in Grgic [15] & $\begin{array}{l}\text { - Yo-Yo test performance in Grgic } \\
\text { et al. [16] }\end{array}$ \\
\hline \multicolumn{3}{|c|}{ Meta-analyses that did not find significant ergogenic effects of sodium bicarbonate } \\
\hline Low & $\begin{array}{l}\text { - General mean power in Carr et al. [13] } \\
\text { - Repeated-sprint ability (total work) in Lopes-Silva et al. [18] } \\
\text { - Anaerobic power in the Wingate test using single-dose supplementation protocol in Lopes- } \\
\text { Silva et al. [19] }\end{array}$ & / \\
\hline Moderate & $\begin{array}{l}\text { - Repeated-sprint ability (best sprint) in Lopes-Silva et al. [18] } \\
\text { - Repeated-sprint ability (last sprint) in Lopes-Silva et al. [18] }\end{array}$ & - Muscle strength in Grgic et al. [17] \\
\hline
\end{tabular}

a greater performance-enhancing effect of multi-day protocol was found on the second and third testing days. Due to the paucity of research, future work on this topic is needed. Future work is also needed to explore the effects of long-term sodium bicarbonate supplementation on different exercise performance outcomes, given that only a handful of studies [4750] have explored this thus far.

Meta-analyses are commonly used to overcome the limitations of small sample sizes in primary studies. However, whether this is achieved or not depends on the pooled sample size. Given their relatively small sizes of pooled sample, some of the analyses included in this review might not have provided definitive answers regarding the effects of sodium bicarbonate supplementation on exercise performance. Specifically, one meta-analysis did not find significant differences between the effects of sodium bicarbonate and placebo in three measures of repeated-sprint performance [18]. In the analysis for total work, the effects favored sodium bicarbonate (pooled effect size: 0.43 ; 95\% CI: $-0.11,0.97$ ), but the difference compared to placebo was not statistically significant $(p=0.12)$. The lack of statistical significance in this analysis could be attributed to the fact that only three studies with a relatively small pooled sample size $(n=27)$ were included, hence making results susceptible to type II error. Therefore, non-significant results of this analysis might not necessarily reflect the absence of an effect in the population. Due to the limited number of primary studies, future research should explore

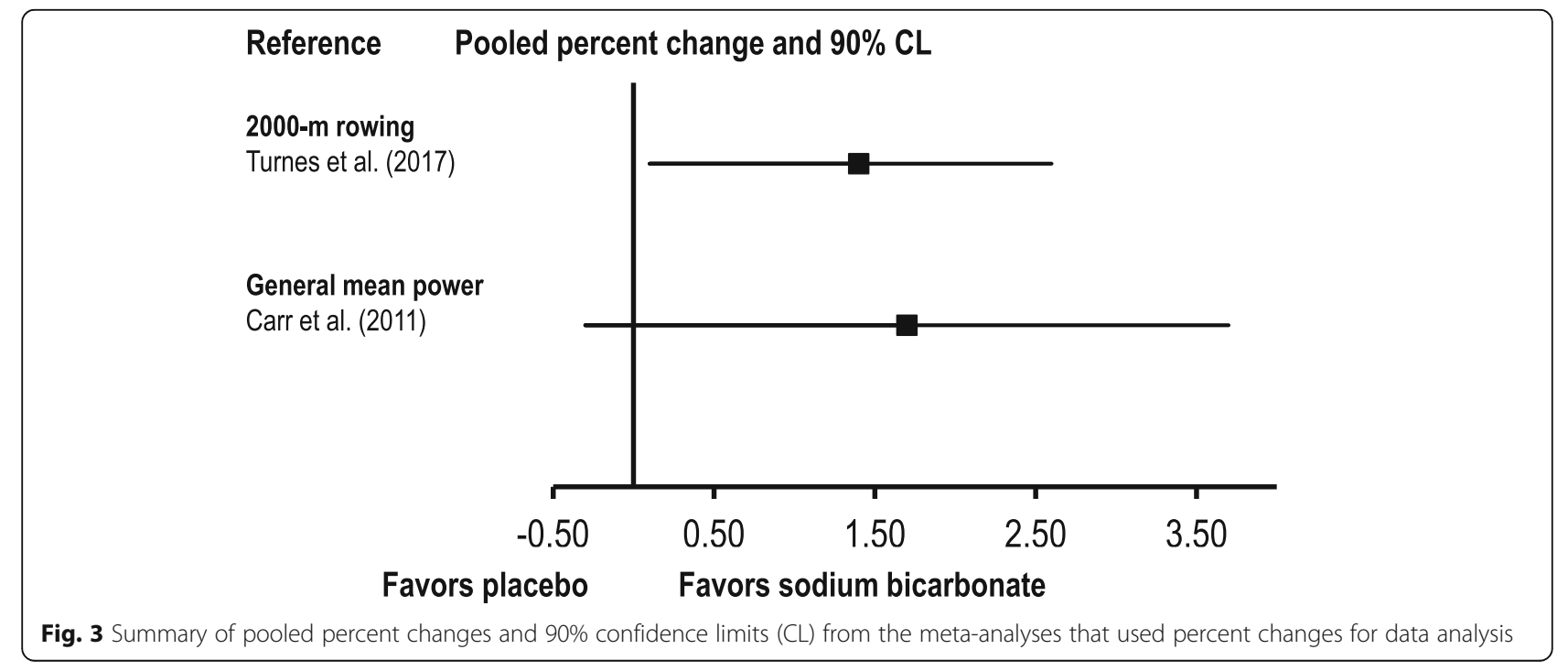


Table 5 Findings of subgroup analyses reported in the included reviews

\begin{tabular}{|c|c|c|}
\hline Reference & Subgroup analyses focus & Subgroups analyses results \\
\hline \multirow[t]{6}{*}{ Carr et al. [13] } & Increase in dose by $1 \mathrm{mmoL} / \mathrm{kg} / \mathrm{body}$ mass & $0.5 \%(90 \% \mathrm{CL}:-0.1,0.6 \%)$ \\
\hline & Five extra bouts & $0.6 \%$ (90\% CL: $0.2,1.0 \%)$ \\
\hline & $10 \times$ duration & $-0.6 \%(90 \% \mathrm{CL}:-1.2,0.3 \%)$ \\
\hline & Non-athletes & $-1.1 \%(90 \% \mathrm{CL}:-2.2,0.0 \%)$ \\
\hline & Females & $-0.7 \%(90 \% \mathrm{CL}:-2.1,0.7 \%)$ \\
\hline & Non-blinded & $0.2 \%(90 \% \mathrm{CL}:-0.5,0.9 \%)$ \\
\hline \multirow[t]{4}{*}{ Grgic et al. [17] - muscle endurance } & Large muscle groups & ES: 0.40 (95\% Cl: $0.13,0.66)$ \\
\hline & Small muscle groups & ES: 0.31 (95\% Cl: 0.04, 0.59) \\
\hline & One time point of ingestion & ES: 0.53 (95\% Cl: $0.14,0.93)$ \\
\hline & Multiple time points of ingestion & ES: 0.23 (95\% Cl: 0.05, 0.42) \\
\hline \multirow[t]{4}{*}{ Grgic et al. [17] - muscle strength } & Tested in a rested state & ES: $0.02(95 \% \mathrm{Cl}:-0.09,0.13)$ \\
\hline & Tested in a fatigued state & ES: $-0.16(95 \% \mathrm{Cl}:-0.59,0.28)$ \\
\hline & One time point of ingestion & ES: $-0.14(95 \% \mathrm{Cl}:-0.50,0.21)$ \\
\hline & Multiple time points of ingestion & ES: 0.04 (95\% Cl: $-0.06,0.14)$ \\
\hline \multirow[t]{7}{*}{ Lopes-Silva et al. [19] - peak power } & Acute ingestion: Wingate bout 1 & ES: $-0.07(95 \% \mathrm{Cl}:-0.36,0.23)$ \\
\hline & Acute ingestion: Wingate bout 2 & ES: $0.00(95 \% \mathrm{Cl}:-0.42,0.42)$ \\
\hline & Acute ingestion: Wingate bout 3 & ES: $0.14(95 \% \mathrm{Cl}:-0.28,0.56)$ \\
\hline & Multi-day ingestion: Wingate bout 1 & ES: 0.79 (95\% Cl: 0.20, 1.37) \\
\hline & Multi-day ingestion: Wingate bout 2 & ES: $1.52(95 \%$ Cl: $0.90,2.13)$ \\
\hline & Multi-day ingestion: Wingate bout 3 & ES: 0.89 (95\% Cl: 0.33, 1.45) \\
\hline & Multi-day ingestion: Wingate bout 4 & ES: 1.36 (95\% Cl: 0.76, 1.96) \\
\hline \multirow[t]{7}{*}{ Lopes-Silva et al. [19] - mean power } & Acute ingestion: Wingate bout 1 & ES: $0.12(95 \%$ Cl: $-0.18,0.41)$ \\
\hline & Acute ingestion: Wingate bout 2 & ES: 0.14 (95\% Cl: $-0.28,0.56)$ \\
\hline & Acute ingestion: Wingate bout 3 & ES: 0.22 (95\% Cl: $-0.20,0.65)$ \\
\hline & Multi-day ingestion: Wingate bout 1 & ES: 0.91 (95\% Cl: 0.43, 1.39) \\
\hline & Multi-day ingestion: Wingate bout 2 & ES: 1.04 (95\% Cl: 0.46, 1.62) \\
\hline & Multi-day ingestion: Wingate bout 3 & ES: 1.66 (95\% Cl: 0.95, 2.38) \\
\hline & Multi-day ingestion: Wingate bout 4 & ES: 2.09 (95\% Cl: 1.31, 2.87) \\
\hline
\end{tabular}

ES: effect size; $\mathrm{CL}$ : confidence limit; $\mathrm{Cl}$ : confidence interval

the effects of sodium bicarbonate supplementation on different measures of repeated-sprint performance.

\section{Methodological quality}

All included reviews were classified as being of moderate or high quality. Therefore, the findings presented in this umbrella review are not confounded by low methodological quality of included reviews. Nevertheless, there are some limitations noted on the AMSTAR 2 checklist that should be considered for future reviews on the topic. For example, none of the included reviews received a point on item 7, which refers to reporting of excluded studies. Future reviews should consider adding a list of excluded studies and provide reasons for their exclusion. This will make the results of the study selection process more transparent and easier to verify. Only one review [14] reported funding sources for the included studies and received a point on item 10. The review noted that none of the included primary studies received funding from sources that might have had a potential commercial interest. Future reviews and primary studies should present information on received funding, as it has been shown that findings of nutrition-related research may be biased in favor of sponsors' products [51].

\section{Conclusion}

Based on meta-analyses of moderate to high quality, it can be concluded that sodium bicarbonate supplementation acutely enhances peak anaerobic power, anaerobic capacity, performance in endurance events lasting $\sim 45 \mathrm{~s}$ to $8 \mathrm{~min}$, muscle endurance, $2000-\mathrm{m}$ rowing performance, and high-intensity intermittent running. The 
ergogenic effects ranged from trivial (pooled effect size: 0.09 ) to large (pooled effect size: 1.26). Still, for most outcomes, sodium bicarbonate elicited comparable ergogenic effects. For example, sodium bicarbonate produced similar effects on performance in endurance events lasting $\sim 5 \mathrm{~s}$ to $8 \mathrm{~min}$, muscle endurance tests, and Yo-Yo test (pooled effect size range: 0.36 to 0.40 ). The quality of evidence presented in the included meta-analyses ranged from low to moderate. More research is needed among women to improve the generalizability of findings.

\section{Abbreviations \\ AMSTAR 2: Assessing the Methodological Quality of Systematic Reviews 2; Cl: Confidence Interval; GRADE: Grading of Recommendations Assessment, Development, and Evaluation; IOC: International Olympic Committee; PICO: Participant-Intervention-Comparison-Outcome}

\section{Acknowledgements}

None.

\section{Authors' contributions}

JG conceived the idea and conceptualized the review. JG and IG conducted the study selection, data extraction, and methodological quality assessment. JG drafted the initial manuscript. JG, IG, JDC, BJS, and ZP contributed to writing the manuscript. All authors read and approved the final manuscript.

\section{Funding}

None.

\section{Availability of data and materials}

Not applicable.

\section{Declarations}

Ethical approval and consent to participate

Not applicable.

\section{Consent for publication}

Not applicable.

\section{Competing interests}

None.

\section{Author details}

${ }^{1}$ Institute for Health and Sport, Victoria University, Melbourne, Australia. ${ }^{2}$ County Hospital Schrobenhausen, Schrobenhausen, Germany. ${ }^{3}$ Centre for Sport Studies, Rey Juan Carlos University, Fuenlabrada, Spain. ${ }^{4}$ Department of Health Sciences, Lehman College, Bronx, NY, USA.

Received: 18 January 2021 Accepted: 3 November 2021

Published online: 18 November 2021

\section{References}

1. Dennig H, Talbott JH, Edwards HT, Dill DB. Effect of acidosis and alkalosis upon capacity for work. J Clin Invest. 1931;9(4):601-13. https://doi.org/10.11 72/JCl100324.

2. Lancha Junior AH, Painelli Vde S, Saunders B, et al. Nutritional strategies to modulate intracellular and extracellular buffering capacity during highintensity exercise. Sports Med. 2015;45(Suppl 1):S71-81. https://doi.org/10.1 007/s40279-015-0397-5.

3. Artioli GG, Gualano B, Coelho DF, Benatti FB, Gailey AW, Lancha AH. Does sodium-bicarbonate ingestion improve simulated judo performance? Int J Sport Nutr Exerc Metab. 2007;17(2):206-17. https://doi.org/10.1123/ijsnem.1 7.2.206.

4. Lindh AM, Peyrebrune MC, Ingham SA, Bailey D, Folland J. Sodium bicarbonate improves swimming performance. Int I Sports Med. 2008;29(6): 519-23. https://doi.org/10.1055/s-2007-989228.
5. Siegler JC, Hirscher K. Sodium bicarbonate ingestion and boxing performance. J Strength Cond Res. 2010;24(1):103-8. https://doi.org/10.151 9/JSC.0b013e3181a392b2.

6. Carr BM, Webster MJ, Boyd JC, Hudson GM, Scheett TP. Sodium bicarbonate supplementation improves hypertrophy-type resistance exercise performance. Eur J Appl Physiol. 2013;113(3):743-52. https://doi.org/10.1007/ s00421-012-2484-8.

7. McNaughton LR, Cedaro R. The effect of sodium bicarbonate on rowing ergometer performance in elite rowers. Aust J Sci Med Sport. 1991;23(3):669.

8. Goldfinch J, McNaugton L, Davies P. Induced metabolic acidosis and its effects on 400-m racing time. Eur J Appl Physiol. 1988;57(1):45-8. https://doi. org/10.1007/BF00691236.

9. McCartney N, Heigenhauser GJ, Jones NL. Effects of $\mathrm{pH}$ on maximal power output and fatigue during short-term dynamic exercise. J Appl Physiol Respir Environ Exerc Physiol. 1983;55(1 Pt 1):225-9. https://doi.org/10.1152/ja ppl.1983.55.1.225.

10. Ducker KJ, Dawson B, Wallman KE. Effect of beta alanine and sodium bicarbonate supplementation on repeated-sprint performance. J Strength Cond Res. 2013;27(12):3450-60. https://doi.org/10.1519/JSC.0b013e3182 $8 f d 310$.

11. Higgins MF, Wilson S, Hill C, Price MJ, Duncan M, Tallis J. Evaluating the effects of caffeine and sodium bicarbonate, ingested individually or in combination, and a taste-matched placebo on high-intensity cycling capacity in healthy males. Appl Physiol Nutr Metab. 2016;41(4):354-61. https://doi.org/10.1139/apnm-2015-0371.

12. Maughan RJ, Burke LM, Dvorak J, Larson-Meyer DE, Peeling P, Phillips SM, et al. IOC consensus statement: dietary supplements and the highperformance athlete. Br J Sports Med. 2018;52(7):439-55. https://doi.org/1 0.1136/bjsports-2018-099027.

13. Carr AJ, Hopkins WG, Gore CJ. Effects of acute alkalosis and acidosis on performance: a meta-analysis. Sports Med. 2011;41(10):801-14. https://doi. org/10.2165/11591440-000000000-00000.

14. Christensen PM, Shirai Y, Ritz C, et al. Caffeine and bicarbonate for speed. A meta-analysis of legal supplements potential for improving intense endurance exercise performance. Front Physiol. 2017:8:240.

15. Grgic J. Effects of sodium bicarbonate ingestion on measures of Wingate test performance: a meta-analysis. J Am Coll Nutr. 2020:1-10. https://doi. org/10.1080/07315724.2020.1850370.

16. Grgic J, Garofolini A, Pickering C, Duncan MJ, Tinsley GM, del Coso J. Isolated effects of caffeine and sodium bicarbonate ingestion on performance in the Yo-Yo test: a systematic review and meta-analysis. J Sci Med Sport. 2020;23(1):41-7. https://doi.org/10.1016/j.jsams.2019.08.016.

17. Grgic J, Rodriguez RF, Garofolini A, Saunders B, Bishop DJ, Schoenfeld BJ, et al. Effects of sodium bicarbonate supplementation on muscular strength and endurance: a systematic review and meta-analysis. Sports Med. 2020; 50(7):1361-75. https://doi.org/10.1007/s40279-020-01275-y.

18. Lopes-Silva JP, Choo HC, Franchini E, Abbiss CR. Isolated ingestion of caffeine and sodium bicarbonate on repeated sprint performance: systematic review and meta-analysis. J Sci Med Sport. 2019;22(8):962-72. https://doi.org/10.1016/j.jsams.2019.03.007.

19. Lopes-Silva JP, Reale R, Franchini E. Acute and chronic effect of sodium bicarbonate ingestion on Wingate test performance: a systematic review and meta-analysis. J Sports Sci. 2019;37(7):762-71. https://doi.org/10.1080/02 640414.2018.1524739.

20. Turnes T, Cruz RSO, Caputo F, de Aguiar RA. The impact of preconditioning strategies designed to improve 2000-m rowing ergometer performance in trained rowers: a systematic review and meta-analysis. Int J Sports Physiol Perform. 2019;14(7):871-9. https://doi.org/10.1123/ijspp.2019-0247.

21. Ioannidis J. Next-generation systematic reviews: prospective meta-analysis, individual-level data, networks and umbrella reviews. Br J Sports Med. 2017; 51(20):1456-8. https://doi.org/10.1136/bjsports-2017-097621.

22. Aromataris $E$, Fernandez $R$, Godfrey $C M$, Holly $C$, Khalil $H$, Tungpunkom $P$. Summarizing systematic reviews: methodological development, conduct and reporting of an umbrella review approach. Int J Evid Based Healthc. 2015:13(3):132-40. https://doi.org/10.1097/XEB.0000000000000055.

23. Fusar-Poli P, Radua J. Ten simple rules for conducting umbrella reviews. Evid Based Ment Health. 2018;21(3):95-100. https://doi.org/10.1136/ebmental-201 8-300014

24. Cohen J. A power primer. Psychol Bull. 1992;112(1):155-9. https://doi.org/1 0.1037/0033-2909.112.1.155. 
25. Shea BJ, Reeves BC, Wells G, Thuku M, Hamel C, Moran J, et al. AMSTAR 2: a critical appraisal tool for systematic reviews that include randomised or non-randomised studies of healthcare interventions, or both. BMJ. 2017;358: j4008. https://doi.org/10.1136/bmj.j4008.

26. Grgic J, Grgic I, Pickering C, Schoenfeld BJ, Bishop DJ, Pedisic Z. Wake up and smell the coffee: caffeine supplementation and exercise performance-an umbrella review of 21 published meta-analyses. $\mathrm{Br} \mathrm{J}$ Sports Med. 2020;54(11):681-8. https://doi.org/10.1136/bjsports-2018-1002 78.

27. Guyatt G, Oxman AD, Akl EA, Kunz R, Vist G, Brozek J, et al. GRADE guidelines: 1. Introduction-GRADE evidence profiles and summary of findings tables. J Clin Epidemiol. 2011;64(4):383-94. https://doi.org/10.1016/j. jclinepi.2010.04.026.

28. Hadzic M, Eckstein ML, Schugardt M. The impact of sodium bicarbonate on performance in response to exercise duration in athletes: a systematic review. J Sports Sci Med. 2019;18(2):271-81.

29. Kalytczak MM, Barros BM, Battazza RA, Bocalini DS, Biasotto-Gonzalez DA, Figueira Junior AJ, et al. The effect of sodium bicarbonate supplementation on electromyographic muscle activity in healthy, physically active individuals: a systematic review. J Diet Suppl. 2020;18(3):334-50. https://doi. org/10.1080/19390211.2020.1766633.

30. Martínez-Rodríguez A, Cuestas-Calero BJ, Hernández-García M, MartíezOlcina M, Vicente-Martínez M, Rubio-Arias JÁ. Effect of supplements on endurance exercise in the older population: systematic review. Int J Environ Res Public Health. 2020;17(14):5224. https://doi.org/10.3390/ijerph17145224.

31. Matson LG, Tran ZV. Effects of sodium bicarbonate ingestion on anaerobic performance: a meta-analytic review. Int J Sport Nutr. 1993;3(1):2-28. https:// doi.org/10.1123/ijsn.3.1.2

32. Peart DJ, Siegler JC, Vince RV. Practical recommendations for coaches and athletes: a meta-analysis of sodium bicarbonate use for athletic performance. J Strength Cond Res. 2012;26(7):1975-83. https://doi.org/10.1 519/JSC.0b013e3182576f3d.

33. Schubert MM, Astorino TA. A systematic review of the efficacy of ergogenic aids for improving running performance. J Strength Cond Res. 2013;27(6): 1699-707. https://doi.org/10.1519/JSC.0b013e31826cad24.

34. Vicente-Salar N, Santos-Sánchez G, Roche E. Nutritional ergogenic aids in racquet sports: a systematic review. Nutrients. 2020;12(9):E2842. https://doi. org/10.3390/nu12092842.

35. Bishop D, Edge J, Davis C, Goodman C. Induced metabolic alkalosis affects muscle metabolism and repeated-sprint ability. Med Sci Sports Exerc. 2004; 36(5):807-13. https://doi.org/10.1249/01.mss.0000126392.20025.17.

36. McNaughton LR, Ford S, Newbold C. Effect of sodium bicarbonate ingestion on high intensity exercise in moderately trained women. J Strength Cond Res. 1997;11(2):98-102.

37. Bishop D, Claudius B. Effects of induced metabolic alkalosis on prolonged intermittent-sprint performance. Med Sci Sports Exerc. 2005;37(5):759-67. https://doi.org/10.1249/01.MSS.0000161803.44656.3C.

38. Delextrat A, Mackessy S, Arceo-Rendon L, Scanlan A, Ramsbottom R, CallejaGonzalez J. Effects of three-day serial sodium bicarbonate loading on performance and physiological parameters during a simulated basketball test in female university players. Int J Sport Nutr Exerc Metab. 2018;28(5): 547-52. https://doi.org/10.1123/ijsnem.2017-0353.

39. Fitts $\mathrm{RH}$. The role of acidosis in fatigue: pro perspective. Med Sci Sports Exerc. 2016;48(11):2335-8. https://doi.org/10.1249/MSS.0000000000001043.

40. Debold EP, Fitts RH, Sundberg CW, Nosek TM. Muscle fatigue from the perspective of a single crossbridge. Med Sci Sports Exerc. 2016;48(11):227080. https://doi.org/10.1249/MSS.0000000000001047.

41. Grgic J, Pedisic Z, Saunders B, Artioli GG, Schoenfeld BJ, McKenna MJ, et al. International Society of Sports Nutrition position stand: sodium bicarbonate and exercise performance. J Int Soc Sports Nutr. 2021;18(1):61. https://doi. org/10.1186/s12970-021-00458-w.

42. Douroudos II, Fatouros IG, Gourgoulis V, et al. Dose-related effects of prolonged $\mathrm{NaHCO} 3$ ingestion during high-intensity exercise. Med Sci Sports Exerc. 2006;38(10):1746-53. https://doi.org/10.1249/01.mss.0000230210. 60957.67.

43. Oliveira LF, de Salles PV, Nemezio K, et al. Chronic lactate supplementation does not improve blood buffering capacity and repeated high-intensity exercise. Scand J Med Sci Sports. 2017;27(11):1231-9. https://doi.org/1 $0.1111 /$ sms. 12792
44. Tobias G, Benatti FB, de Salles PV, et al. Additive effects of beta-alanine and sodium bicarbonate on upper-body intermittent performance. Amino Acids. 2013;45(2):309-17. https://doi.org/10.1007/s00726-013-1495-z.

45. Driller MW, Gregory JR, Williams AD, Fell JW. The effects of serial and acute NaHCO3 loading in well-trained cyclists. J Strength Cond Res. 2012;26(10): 2791-7. https://doi.org/10.1519/JSC.0b013e318241e18a.

46. McNaughton L, Thompson D. Acute versus chronic sodium bicarbonate ingestion and anaerobic work and power output. J Sports Med Phys Fitness. 2001;41(4):456-62.

47. Edge J, Bishop D, Goodman C. Effects of chronic NaHCO3 ingestion during interval training on changes to muscle buffer capacity, metabolism, and short-term endurance performance. J Appl Physiol. 2006;101(3):918-25. https://doi.org/10.1152/japplphysiol.01534.2005.

48. Driller MW, Gregory JR, Williams AD, Fell JW. The effects of chronic sodium bicarbonate ingestion and interval training in highly trained rowers. Int J Sport Nutr Exerc Metab. 2013;23(1):40-7. https://doi.org/10.1123/ ijsnem.23.1.40.

49. Siegler JC, Marshall PWM, Finn H, Cross R, Mudie K. Acute attenuation of fatigue after sodium bicarbonate supplementation does not manifest into greater training adaptations after 10-weeks of resistance training exercise. PLoS One. 2018;13(5):e0196677. https://doi.org/10.1371/journal.pone.01 96677.

50. Wang J, Qiu J, Yi L, Hou Z, Benardot D, Cao W. Effect of sodium bicarbonate ingestion during 6 weeks of HIIT on anaerobic performance of college students. J Int Soc Sports Nutr. 2019;16(1):18. https://doi.org/10.1186/s12970019-0285-8.

51. Lesser LI, Ebbeling CB, Goozner M, Wypij D, Ludwig DS. Relationship between funding source and conclusion among nutrition-related scientific articles. PLoS Med. 2007;4(1):e5. https://doi.org/10.1371/journal.pmed.004 0005.

\section{Publisher's Note}

Springer Nature remains neutral with regard to jurisdictional claims in published maps and institutional affiliations.

Ready to submit your research? Choose BMC and benefit from:

- fast, convenient online submission

- thorough peer review by experienced researchers in your field

- rapid publication on acceptance

- support for research data, including large and complex data types

- gold Open Access which fosters wider collaboration and increased citations

- maximum visibility for your research: over $100 \mathrm{M}$ website views per year

At BMC, research is always in progress.

Learn more biomedcentral.com/submissions 